\title{
CONOCIMIENTO HUMANO Y CONOCIMIENTO
} ANIMAL

\author{
José Ignacio Murillo \\ Universidad de Navarra
}

Resumen: Es común en el pensamiento actual la negación o incluso la falta de percepción de una posible diferencia esencial entre el conocimiento humano y el animal. En este texto se presentan algunas razones de esta situación, se plantea si es adecuado usar el término conocimiento tanto para los hombres como para el resto de los animales $y$, desde una perspectiva aristotélica, se recupera un debate de la antropología del siglo pasado en el que se pone de manifiesto un aspecto de la cuestión frecuentemente pasado por alto en nuestra época.

Palabras clave: conocimiento animal, inteligencia, antropología, Köhler, Pavlov, Scheler, Aristóteles.

\section{Human knowledge and animal knowledge}

Abstract: In contemporary thinking it is common the negation or even the incapacity of perceiving an essential difference between human and animal cognition. In this text, some reasons that explain this situation are offered, the problem of the opportunity of using the same term for animal and human cognition is revised and, from an Aristotelian perspective, a debate of last century philosophical anthropology is revisited that addresses an aspect of this problem frequently ignored.

Keywords: animal cognition, intelligence, anthropology, Köhler, Pavlov, Scheler, Aristotle.

Recibido: 29/08/2017 Aprobado: 5/11/2017 
José Ignacio Murillo

\section{La auestión de la distinción entre el hombre y el animal en la actualidad}

Uno de los criterios para comprender una cultura es detenerse en lo que podríamos denominar sus "puntos ciegos", es decir, aquellas distinciones que le resulta difícil percibir o que tiende a considerar irrelevantes. Desde este punto de vista, nuestra cultura parece abocada a percibir cada vez de manera más borrosa cualquier distinción tajante entre el hombre y resto de los animales, al menos alguna que debiera empujar —más allá de la mera decisión arbitraria - a tratar al ser humano de un modo distinto respecto de aquellos. $¿$ Es este el fruto de un aumento de nuestro conocimiento y experiencia de la realidad o se debe tan solo a una incapacidad para advertir algo que, sin embargo, debería resultar claro?

Para muchos de nuestros antepasados esta omisión parecería sorprendente. Quizá, como consecuencia del modo en que hoy nos percibimos a nosotros mismos, tendemos a pensar que aquellos se sentían cómodamente inmersos en la naturaleza, sin plantear más interrogantes, hasta que llegaron personajes como los filósofos, que insistieron en que el hombre era distinto porque solo él era capaz de hacer filosofía, o novedosas religiones, como el judaísmo o el cristianismo, que consideraban a los seres humanos como los destinatarios de una elección que los elevaba por encima del resto de los seres hasta situarlos en el centro de la realidad. Sin embargo, los datos de que disponemos acerca de las culturas pretéritas parecen abonar la idea de que el 
hombre siempre se ha considerado un ser distinto y dotado de algún extraordinario privilegio respecto del resto de los animales (Brague, 2013: 14 ss.).

Para la teoría que pugna actualmente por volverse dominante esa conciencia, si ha existido, solo sería una forma de especismo, es decir, la expresión de una tendencia innata al egoísmo contra la que sería preciso luchar para hacer espacio a las otras especies en el ámbito de los seres dotados de dignidad. Resulta curioso, de todos modos, que esta postura considere irrelevante el hecho de que solo el hombre se preocupe por tales inquietudes morales y que, aun concediendo tanta importancia a la moral — pues no solo la invoca sino que exige respetarla a todos sus congéneres-, considere poco relevante a la hora de establecer distinciones el hecho de poder reconocerla.

En esta ocasión no me voy a detener a analizar esta paradoja, sino otra cuestión que, a mi modo de ver, resulta todavía más básica: el examen de las posibles diferencias entre el conocimiento humano y el del resto de los animales. No pretendo llevar a cabo un planteamiento exhaustivo o sistemático del tema, sino solo considerar algunas diferencias e intentar ponerles nombre, dejando en gran medida al lector la tarea de valorar su alcance. Avanzo que la tesis que voy a defender, contra muchos de mis contemporáneos, es que, al menos si nos debemos apoyar en los datos que nos ofrecen la etología y la psicología comparada, existe una radical diferencia entre el conocimiento humano y el del resto de los animales.

De todos modos, tampoco me propongo en estas páginas probarla exhaustivamente. Como no dispongo de espacio para detenerme en toda la literatura que existe sobre el tema, me concentraré en identificar algunas de 
José Ignacio Murillo

las razones intelectuales que explican la dificultad para ver la diferencia, que se traducen también en dificultades para interpretar los datos de que disponemos. A continuación intentaré plantear el problema desde una perspectiva aristotélica, no sin antes abordar alguna de las dificultades que esta plantea. En este contexto, me propongo retomar un curioso episodio de la historia del debate acerca de la inteligencia animal para, desde él, señalar algunas características distintivas del conocimiento humano con la esperanza de que puedan arrojar luces sobre observaciones empíricas con que se busca aclarar el problema.

\section{Algunas razones intelectuales que difialtan adarar la auestión}

$\mathrm{El}$ primer factor que ha influido en la aproximación u homologación del conocimiento humano al animal es el creciente influjo del empirismo en nuestra concepción del conocimiento. El empirismo defiende que todo nuestro conocimiento se reduce a los sentidos. Puesto que compartimos en gran medida nuestra dotación sensorial con los animales, esta afirmación conduce necesariamente a diluir la barrera entre lo que los animales pueden conocer y lo que puede conocer el hombre. El éxito del empirismo se encuentra muy vinculado al de la ciencia moderna. Es cierto que la nueva ciencia se apoya en la matemática, cuya conexión con la experiencia sensible es todavía hoy objeto de debate, pero lo característico de la nueva ciencia es que toda hipótesis o conjetura solo puede adquirir legitimidad en la medida en que se contrasta con la experiencia sensible. El recurso a la experimentación y a la observación controlada es central tanto en la física como en las ciencias de la vida y en 
todas aquellas disciplinas que aspiren a un reconocimiento teórico como el suyo. Este éxito ha avalado la idea de que el único punto de contacto de nuestra mente con la realidad es la experiencia sensible.

Un segundo factor relevante es el afianzamiento en la cultura científica del naturalismo y, en particular, del naturalismo evolucionista. Según este paradigma, existe una continuidad de fondo entre todos los seres vivos $y$, puesto que unos proceden de otros, no puede haber nada en los posteriores que exceda las potencialidades de la materia de que todos ellos están compuestos. La tesis darwinista de que los cambios son progresivos y casi imperceptibles abona la idea de que las diferencias que parecen grandes son solo el fruto de una ilusión y pueden ser reducidas a pequeños cambios que han tenido lugar a lo largo de largos periodos de tiempo. Lo que denominamos inteligencia no escapa de este paradigma, y nos sentimos, por tanto, obligados a explicar una inteligencia como la humana sin recurrir a grandes saltos, pues aceptarlos sería lo mismo que aceptar nuestra ignorancia. Es cierto que términos como salto o emergencia siguen presentes en el discurso científico o filosófico, pero, al usarlos, es difícil escapar a la mala conciencia de que, sin más, se deja algo importante por explicar.

Un tercer factor proviene en particular del ámbito de la filosofía. Se trata del giro lingüístico que se ha operado en la filosofía en el último siglo. Hasta el idealismo, los filósofos hablaban del entendimiento o de la razón y sus conceptos e ideas. Pero seguramente la reacción positivista que siguió a este condujo a dirigir la mirada hacia hechos más patentes y controlables, y así se fue fraguando el interés por los signos, el lenguaje —es el nacimiento de la 
José Ignacio Murillo

filología- y las formulaciones lógicas del pensamiento, que tienden a convertirlo en claro y controlable. El resultado es una fuerte tendencia a identificar el pensamiento con el lenguaje. Un intento que ha adoptado tantas formas como formas existen de considerar el lenguaje, desde las que conceden prioridad al lenguaje científico entendido como paradigma de rigor intelectual hasta las que parten del lenguaje ordinario, que pone de manifiesto nuestra primaria instalación en el mundo y el sentido y las diversas facetas de la experiencia de los seres humano.

$\mathrm{Y}$ esto esta relacionado con un cuarto factor. El lenguaje era visto desde antiguo no como un órgano teórico sino como un órgano práctico, cuya primera intención no era el saber sino la comunicación, y que además no estaba forjado para transmitir el conocimiento teórico, sino sobre todo para las exigencias de la vida común. En este sentido, hablar es una acción que pretende transformar la realidad. ¿Por qué no entender el entero conocimiento humano como un modo de intervenir en ella? Se trata de una tesis que, con todos los respetos para las distintas tradiciones que se identifican con ese nombre, podríamos denominar pragmatista: el conocimiento es una acción transformadora, una de las formas posibles de intercambio con el mundo, que mide su valor por su eficacia. Esta tesis lleva a una transformación de la noción de verdad que, si se mantiene, solo puede definirse por relación a alguna forma de éxito ajeno al saber mismo.

Nos encontramos de nuevo ante una postura que limita cualquier intento de trazar un límite entre el ser humano y el resto de los animales y que se encuentra conectado con otro de los rasgos de nuestra situación cultural: la 
pérdida de interés por el momento contemplativo o teórico del conocimiento, del interés por la verdad en cuanto tal y por su conocimiento. Por eso no debe extrañar que las definiciones contemporáneas de la inteligencia la reduzcan más o menos a algunos de los aspectos de lo que los antiguos denominaban logos o razón y quede así caracterizada tan solo como una simple capacidad de resolver problemas.

\section{3. ¿Es legítimo usar el término conocimiento tanto para el hombre como para el animal?}

Es este, sin embargo, el elemento central de la perspectiva aristotélica. E1 hombre se caracteriza por su deseo de saber, es decir, por ser capaz de proponerse como fin - el mejor de aquellos a los que cabe aspirar- la contemplación de la verdad (Aristóteles). De todos modos, el recurso a Aristóteles en este contexto no deja tampoco de arrojar sospechas, pues en realidad algunos de sus planteamientos parecen abonar la confusión entre las formas de conocimiento específicamente humanas y las de los animales.

Una de las dificultades deriva precisamente de la insistencia de la tradición aristotélica por atribuir el término conocimiento o cognición tanto a los animales como a los seres humanos. En efecto, el conocimiento se presenta como una actividad vital que podemos encontrar tanto en nosotros como en los gusanos y los caracoles. Si Aristóteles estuviera seguro de que existe un abismo entre el conocimiento humano y el animal, ¿por qué usar el mismo término para ambos y convertirlos, a lo sumo, en especies del mismo género? En este sentido, si se quiere sostener la radical distinción entre el hombre y el 
José Ignacio Murillo

animal, parece más consecuente la actitud de los autores mecanicistas, que distinguen netamente entre un espíritu dotado de consciencia y de verdadero saber, por oposición al resto de los animales, cuyos movimientos pueden ser explicados en términos meramente mecánicos.

La pregunta que debe ser respondida para dirimir este problema es si una distinción — que no solo es de nivel, sino que realmente resulta radicalpuede ser albergada por una misma noción nombrada de idéntico modo. La metafísica clásica respondería a esta dificultad seguramente invocando la analogía, que no duda en aplicar al mismo ente y a distinciones tan radicales como la del ser creado y el increado. Con esta mención pretendo alertar de que bajo nombres semejantes pueden albergarse distinciones importantes, incluso, en el sentido metafísico del término, trascendentales. ¿Resulta esto adecuado o solo un pretexto responsable de que la equivocidad genere confusión?

Para resolver esta cuestión, juzgo importante distinguir entre lo que podemos denominar diferencia y distinción. Las diferencias se encuentran en el plano de las notas de los conceptos mientras que las distinciones remiten directamente a la realidad. En este sentido, el conocimiento animal y el humano pueden ser conocimiento desde una perspectiva común, aunque se trate de realidades radicalmente distintas. No pocos filósofos han intentado contrarrestar la ambigüedad a que puede inducir este uso amplio o analógico de los términos, tan característico del aristotelismo, insistiendo en la necesidad de que los términos que se aplican a las distintas realidades sean, en la 
medida de lo posible, totalmente unívocos. Sin embargo, no son pocas las dificultades que derivan de este intento.

Por una parte, la proliferación de nombres para denominar realidades aparentemente distintas acaba separando excesivamente el lenguaje científico del lenguaje natural haciéndole perder conexión con la experiencia ordinaria. Esto se debe, sobre todo, a que un lenguaje excesivamente unívoco, además de convertirse en prolijo y difícilmente manejables, acaba, sobre todo, por poner en sordina las semejanzas, continuidades y analogías que contiene lo real.

De todos modos, en mi opinión, la justificación de un uso analógico de los términos se encuentra habitualmente, y el conocimiento es uno de estos casos, en el itinerario de nuestro conocimiento, que parte de lo sensible. Para nosotros la más patente forma de conocer es la que nos ofrecen los sentidos y solo desde ellos nos remontamos a nombrar y caracterizar las formas superiores del conocimiento, que, aunque ya estuvieran operantes desde el principio, solo pueden ser caracterizadas por referencia a las primeras.

Pero la posible equivocidad de los términos no solo afecta al aristotelismo. En este el término intelecto o inteligencia (nôेs) quedaba confinado con bastante claridad a las formas humanas y sobrehumanas del conocimiento, a las que podemos llamar con más propiedad con ese nombre. Sin embargo, en la actualidad inteligencia es un término que puede ser usado tanto para el ser humano como para los animales y las máquinas, haciendo de este modo más preocupante la confusión. 
José Ignacio Murillo

Para aclararnos en este bosque, y dado que he anunciado que asumiré e intentaré justificar una perspectiva aristotélica, me limitaré a afirmar que el término conocimiento solo puede ser aplicado con rigor a los seres vivos y que esta es la razón por la que lo he adoptado en mi título. Desde este punto de vista, cabe adoptar un sentido lato del término inteligencia, si la entendemos como capacidad de resolver problemas, hasta el punto de que podemos atribuirla metafóricamente a las máquinas, pero, si entendemos la inteligencia como sinónima del intelecto, esta solo puede ser una forma de conocimiento, en la que, por cierto, se da del modo más pleno y cabal el sentido que se contiene en el término conocimiento.

Usaré, sin embargo, como sinónimos conocimiento y cognición, siguiendo la tradición aristotélica y separándome, en cambio, del uso que a menudo recibe el término en el pensamiento moderno y en las ciencias cognitivas. En nuestro contexto, conocimiento es la capacidad que tienen algunos seres vivos de poseer lo otro como otro, de vivirlo, desde las formas más básicas hasta las más altas, albergándolas en sí mismos sin necesidad de alterarlo o destruirlo, algo que les permite adoptar diversas actitudes y movimientos respecto de ellas. La consecuencia del conocimiento es vivir en un ámbito más amplio que el del propio organismo, abriendo un entorno significativo al que, al menos en el caso del ser humano, podemos denominar mundo.

Siguiendo esta consideración podemos aventurar que tanto el animal como el ser humano conocen, pero que quizá conocer no significa lo mismo para ambos. Y este sería otro modo de aludir a esa característica con que solemos referirnos a los individuos de la especie humana, la de ser personas. 
Ser persona no es solo poseer unas determinadas características, sino una condición que dota al vivir de un significado distinto.

\section{La relevancia de una vieja polémica entre psioólogos: el debate Köhler versus Pavlov en torno a la inteligencia de los primates}

Pero volvamos sobre nuestra pregunta: ¿existe alguna distinción entre el conocimiento humano y el animal que presuponga un salto tan radical que permita oponer al primero frente al segundo? $\mathrm{O}$, dicho de otro modo, ¿existe en el conocimiento humano algo irreductible al conocimiento de los animales? Es esta una pregunta difícil, pues, evidentemente, su respuesta depende del punto de vista que se adopte, y este depende especialmente de qué entendamos por conocer. Por esta razón, me he apresurado a aportar una especie de definición. Me gustaría que fuera suficientemente clara como para servir de criterio para la investigación científica.

De todos modos, no estoy seguro de si todos aquellos que buscan comparar las capacidades cognitivas humanas con las de otras especies tienen claro lo que buscan. Y esto es importante para diseñar, en su caso, los experimentos y, en cualquiera de ellos, para entender lo que observamos. Por eso no acabo de compartir totalmente el optimismo de Tomasello (Tomasello) cuando señala que nos encontramos en una posición mejor que la de nuestros antepasados a la hora de responder esta pregunta. En su opinión, tenemos más conocimientos que nuestros antepasados acerca de las capacidades cognitivas de los primates y de los niños, y esto nos ofrece una notoria ventaja. En la mía, la falta de una definición clara del conocimiento o de la inteligen- 
José Ignacio Murillo

cia, de claridad acerca de lo que buscamos, nos lastra definitivamente. Por otra parte, en ocasiones parece que hemos olvidado que los humanos hemos convivido con los animales y con los niños desde tiempo inmemorial y, aunque esa experiencia no ha sido sometida al método experimental, pienso que no debe ser desdeñada. Desde siempre, hemos sabido que los animales conocen como nosotros hasta cierto punto, pero también que hay algo que para nosotros resulta muy importante en que nos distinguimos, algo de lo que frecuentemente disponemos y que nos lleva a conducir nuestra vida de un modo muy diferente de la suya.

La historia reciente del estudio científico de esta cuestión ha estado frecuentemente guiada por teorías acerca de la naturaleza de la inteligencia humana. Así, por ejemplo, Thorndike (Windholz) intentaba explicar el aprendizaje animal basándose en su teoría del ensayo error, mientras que Köhler, a la luz de sus experimentos, sostenía la existencia de una intuición o comprensión (Einsicht) que permitía a los primates resolver problemas con expedientes nuevos. En ambos casos, su interpretación dependía de su teoría del conocimiento. Otros, como Pavlov, que estudiaba el comportamiento desde la noción de reflejo, atribuían las diferencias entre los primates y los humanos a la falta en los primeros de un sistema de signos de segundo nivel, es decir, de un lenguaje como el humano.

Los nombres mencionados representan una de las etapas de los estudios de psicología comparada, que, en mi opinión, resulta muy ilustrativa. Especial interés revista el debate entre Köhler y Pavlov. A continuación me centraré en un aspecto de este debate, que dio lugar a un famoso experimento 
que ha recibido diversas interpretaciones. Lo presento como un ejemplo de cómo influye en esta cuestión la teoría del conocimiento que se sostiene -o la falta de ella- y como una ocasión para esbozar una propuesta personal acerca del asunto.

Como es bien conocido, Köhler llevó a cabo una serie de experimentos y observaciones sobre el comportamiento de los primates (Köhler). En ellos encontró cierta capacidad de invención que les permitía incluso usar algunos instrumentos y expedientes nuevos para resolver las tareas que se les presentaban. Pavlov, aunque valoraba las aportaciones de Köhler, no compartía sus conclusiones, de modo que acabó por elaborar, años más tarde, sus propios experimentos. Es en este contexto en el que diseñó un famoso experimento que tuvo por protagonista a un chimpancé llamado Raphael.

Raphael aprendió a desplazarse entre unas plataformas que se encontraban separadas por el agua. En una de ellas la comida estaba rodeada de un fuego, que Raphael aprendió a apagar con un vaso que llenaba de un grifo que se encontraba en otra plataforma a la que accedía a través de una pértiga. La situación se complicó para él cuando, para acceder al vaso, tenía que abrir una caja mediante un mecanismo de cierta complejidad. Al mismo tiempo Raphael había aprendido a refrescarse con el agua del estanque sobre el que se hallaban las plataformas. En un determinado momento, se dispuso que el grifo ya no proporcionara agua, para ver cuál era la reacción del primate. Éste, en ninguno de los casos, fue capaz de resolver el obstáculo que se interponía entre él y la comida (Razran). 
José Ignacio Murillo

Para el humano, lo que sorprende de la conducta de Raphael es que se aferrara al procedimiento aprendido sin esforzarse en modo alguno por comprenderlo, sin percatarse de que aquello con que apagaba el fuego estaba ilimitadamente a su disposición.

Desde una perspectiva realista, este experimento ha servido como una prueba de la falta de nociones universales en los primates (Prieto López). Se puede aducir que el hecho de que Raphael no fuera capaz de alcanzarlas no es óbice para que lo hicieran otros ejemplares de su especie. En este sentido, podemos aceptar que el experimento no es definitivamente probatorio. De todos modos, la conducta de los chimpancés y otros primates tal como la conocemos es consistente con la ausencia de esta capacidad que, para nosotros, es decisiva. No solo se encuentra en la base de nuestro lenguaje, sino en nuestro modo más básico de aproximarnos a la realidad. Lo que hubiéramos esperado incluso de un niño de cierta edad es otra conducta. El humano no solo resuelve un problema sino que puede y suele preguntarse por qué son eficaces los expedientes con que lo resuelve. No se trata solo de conseguir el alimento, sino de "apagar el fuego", es más, de comprender que "el agua apaga el fuego" y que esto es así "porque es agua”, es decir, en virtud de lo que es y de las propiedades que por ello le corresponden. Es lo que de un modo no especialmente metafísico podemos denominar "esencia". Se trata de una expresión especialmente cara a Scheler, que llegaba a sostener que la inteligencia orgánicamente determinada se oponía —en un sentido fuerteal espíritu por esta característica (Scheler). 
Siguiendo con esta línea interpretativa, lo que falta al primate es la capacidad de concebir que lo que apaga el fuego es lo mismo que le refresca. Como se puede ver, el vínculo entre ambas situaciones se establece en virtud de lo que hemos llamado la esencia del agua. De todos modos, esto no quiere decir que el humano conozca la esencia del agua, en el sentido de que sepa todo lo que del agua se puede saber. Se trata tan solo de que es capaz de captar "agua" como un contenido nocional consistente que incluye unas deterdeterminadas notas que cabe esperar allá donde de nuevo la encontremos; es decir, que es siempre "lo mismo".

Nos encontramos ante algo distinto de las representaciones que, como está bien probado, puede elaborar y aplicar el animal. El animal puede distinguir categorías de objetos ante los que reacciona y se comporta de modo distinto, y puede incluso repartir objetos entre diversas categorías (Vonk and MacDonald; Diéguez). Pero lo que no parece mostrar es que objetive un contenido mental estable que reúna un grupo de características y que pueda guiar, por tanto, una investigación acerca de las propiedades que corresponden a su referente.

En realidad, lo que echamos de menos en la conducta animal es la capacidad de enfrentarse a un contenido mental, de detenerse ante él. Esto es lo que, por otra parte, denominamos pensar. Como afirma Polo, pensar no es otra cosa que "pararse a pensar". Pero para esto es preciso que captemos el presente. Esta peculiaridad humana se traduce, por otra parte, en que somos los únicos animales que se representan la temporalidad; algo que se manifies- 
José Ignacio Murillo

ta, entre otras cosas, en nuestro interés por medir los movimientos, por medir el tiempo.

Scheler se interesó por los experimentos de Köhler, a los que se refirió en su obra El puesto del hombre en el cosmos, pero no llegó a tiempo — pues murió antes- para conocer el experimento de Pavlov que, con todas sus limitaciones, parecía darle la razón. En cualquier caso, mi impresión es que, casi un siglo después de aquel debate, la atención hacia este aspecto de la cognición humana parece haber desaparecido. Gran parte de la discusión se ha desplazado hacia otros aspectos de la conducta y, en particular, hacia la capacidad de manipular símbolos. Sin embargo, me cuesta encontrar referencias a este aspecto de la cuestión, que me parece central y que, y esto es quizá lo más preocupante, resulta indispensable para hacer ciencia también en el caso de aquellos que parecen pasarlo por alto.

\section{Bibliografia}

Aristóteles, Protréptico: una exhortación a la filosofía, ed. Carlos. Megino Rodríguez, Abada, Madrid, 2006.

R. Brague, Le Propre de L'bomme: Sur Une Légitimité Menacée, Paris, Flammarion, 2013.

A., Diéguez, "Conceptual Thinking in Animals. Some Reflections on Language, Concepts, and Mind”, en Darwin's Evolving Legacy, ed. J. Martínez-Contreras and A. Ponce de León, México, Siglo XXI y Universidad Veracruzana, 2011, pp. 383-395.

W. Köhler, Intelligenzprüfungen an Menschenaffen, Heidelberg, Springer,1963.

L. Prieto López, El hombre y el animal: nuevas fronteras de la antropología, Madrid, Biblioteca de Autores Cristianos, 2008.

G. Razran, "Raphael's 'idealess' behavior", Journal of Comparative and Physiological Psychology, 54.4 (1961), pp. 366-367. 
M. Scheler, El puesto del hombre en el cosmos; la idea de la paz perpetua y el pacifismo, Barcelona, Alba Editorial, 2000.

M. Tomasello, A Natural History of Human Thinking, London, Harvard University Press, 2014.

J. Vonk and S. E MacDonald. "Natural Concepts in a Juvenile Gorilla (Gorilla Gorilla Gorilla) at Three Levels of Abstraction", Journal of the experimental analysis of behavior 78.3 (2002), pp. 315-32.

G. Windholz, "Pavlov vs. Köhler. Pavlov's Little-Known Primate Research." The Pavlovian journal of biological science 19.1, pp. 23-31.

\author{
José Ignacio Murillo \\ jmurillo@unav.es
}


